\title{
A MATLAB-based Design of Shipboard Antenna Gyro Loop System
}

\author{
SUN Linfei
}

China Satellite Maritime Tracking and Control Department, Jiangyin, 214431, China

Key words: FOG; Simulink; MATLAB; gyro loop; shipboard antenna

\begin{abstract}
Fiber optic gyroscope (FOG) plays an important role in shipboard antenna system. The technical specifications of FOG include B.bias and SF. The paper describes in detail the methods and processes of system modeling and performance analysis with MATLAB as well as the stable precision simulation with Simulink. The result of simulation is coincident with the actual test an axis platform . The design indicates that the time of system design and debugging and the risk of project.
\end{abstract}

\section{Introduction}

The biggest difference between the ship antenna and the ground fixed antenna is the antenna base in the waves, Chung and hull power under the joint action of constant changes, which requires the servo system to stabilize the antenna, isolated hull swing, to ensure that the antenna beam pointing stable. The stabilization mode is usually used to mount the gyro on the antenna stabilizing shaft to detect the angular rate change of the hull on the shaft, to feed back the signal back to the calibration circuit, and then to output the corrected signal to the drive device to drive the antenna To move in the direction of the swing, so as to isolate the hull swing.

In this type of control loop system parameter debugging often used trial and error method, debugging cycle is long, and debugging in the intense oscillation process will cause some damage to the transmission chain.

MATLAB software and its environment Simulink simulation tool as the current international control of the most popular system modeling, analysis and simulation software for the stability of the control system design can play a multiplier effect.

In the following, the actual design of a kind of shipborne antenna is taken as an example, and the parameters of gyro loop are optimized by MATLAB software and Simulink simulation tool.

\section{Parameter Index of Fiber Optic Gyroscope}

The original designation of the gyro ring used in a design of a ship-borne antenna is an optical fiber rate gyroscope which converts the angular rate signal into a DC voltage signal proportional to the input angular rate through the angular velocity signal of the sensitive carrier in the axial direction to provide Gyro ring for control. Compared with the mechanical gyroscope, fiber optic gyro has no mechanical movement, start time is short, long life, high precision, dynamic range and so a bit.

1.1. Zero bias

Zero is the amount of gyro output when the input speed is zero in the gyro. The zero bias can be expressed as zero-voltage stability and zero-voltage repetition. Zero-voltage stability refers to the degree of dispersion of the gyro's output around its mean value when the input angular rate is zero, expressed as the standard deviation of the output, or it can be called zero drift, the lower the value, the better the stability The The zero-point voltage repeatability refers to the degree of coincidence between repeated measurements of the gyro zero bias under the same conditions and within the specified interval, expressed as the standard deviation of the zero bias of each test.

1.2. Scale factor

The scale factor refers to the ratio of the gyro output to the input angular rate, expressed as a slope of a particular straight line that is fitted by the least squares method based on the input input output measured over the entire input angular rate range The The unit of scale factor is $\mathrm{mv} /\left({ }^{\circ} / \mathrm{s}\right)$. 
Represents the scale factor of the scale factor performance, etc., the unit commonly used\%.

\section{Tracking loop design}

The antenna is a medium-sized antenna, the antenna surface diameter of $10 \mathrm{~m}$, the use of azimuth - pitch (A-E) -shaped structure, fiber optic rate gyroscope placed on the pitching arm. The control loop consists of a speed loop, a gyro ring, and a position loop.

\subsection{Speed loop}

The speed loop $K^{*} \beta>>1$ ( $\mathrm{K}$ is the speed of the main channel of each channel amplification of the product, $\beta$ for the feedback channel transfer coefficient), then the speed loop closed-loop magnification and the main channel has nothing to do, it is equal to the feedback channel transfer coefficient The countdown. In this way, the main channel power amplifier and the motor's nonlinearity will be overcome. In addition, the speed loop settings can also improve the speed of the servo system.

\subsection{Gyro ring}

The gyro ring is fed back to the gyro ring. The azimuth loop and the pitch loop each use an optical fiber rate gyroscope. One of the gyro's sensitive axes is parallel to the pitch axis and the other is parallel to the azimuth axis (when the pitch angle is equal to zero). When the hull swings to make the antenna pointing change, the gyro will be sensitive to this change and output control information, the control signal through the gyro ring to the opposite direction of rotation with the swing, so as to keep the antenna pointing the same. Its control parameters are to adjust the focus.

\subsection{Position loop}

The position loop completes the position control of the antenna. The tracking receiver outputs the error voltage corresponding to the pointing error angle, which is added to the speed loop by correction, gyro ring correction and correction. The speed loop drives the antenna to rotate and align the antenna axis to the target.

\section{MATLAB software analysis and simulation}

Taking the parameters of the pitch axis gyro as an example, the process of system modeling, design and simulation using MATLAB software and Simulink simulation tool is introduced.

\subsection{System modeling}

The square wave signal is input to the pitch control loop, and the transfer function of the pitch mechanism is determined by the feedback signal.

The transfer function of the control loop (including the position loop, velocity loop and mechanical structure) is simplified to the second order oscillation link:

$$
\mathrm{G}_{0}=\frac{\mathrm{K}_{\mathrm{s}} \omega_{\mathrm{n} 0}^{2}}{1+2 \xi \omega_{\mathrm{n} 0}+\omega_{\mathrm{n} 0}^{2}}
$$

Its overshoot $\mathrm{Mp}$ and the corresponding peak time tp, the formula is:

$$
\begin{gathered}
\mathrm{M}_{\mathrm{p}}=\mathrm{e}^{-\pi \xi \sqrt{1-\xi^{2}}} \\
\mathrm{t}_{\mathrm{p}}=\frac{\pi}{\omega_{\mathrm{n} 0} \sqrt{1-\xi^{2}}}
\end{gathered}
$$

According to the relevant data of the test, we can get: $\mathrm{Ks}=3$; $\mathrm{Mp}=0.03$; tp $=0.275$.

3.2. MATLAB software analysis

Theoretical analysis shows that the transfer function of G0 system to meet: stability accuracy of less than or equal to $0.00725^{\circ}$, isolation needs greater than or equal to $45 \mathrm{~dB}$.

Through the MATLAB software found that the use of pure Ks correction will make the gyro ring oscillation, the oscillation is due to lack of phase margin, it is necessary to compensate for the problem. Take the lead lag correction, which ahead of the network form:

$$
\mathrm{D}_{\mathrm{sd}}=\frac{\mathrm{T}_{\mathrm{d}} \mathrm{s}+1}{\beta \mathrm{T}_{\mathrm{d}} \mathrm{s}+1}
$$

According to the characteristics of G0, it is intended to produce an advanced angle of about $60^{\circ}$ at an angular velocity equal to $9 \mathrm{rad} / \mathrm{s}$. According to formula :: 
You can get $\mathrm{T}_{\mathrm{d}}=0.08$.

$$
\begin{aligned}
& \beta=\frac{1-\sin \varphi}{1+\sin \varphi} \\
& \mathrm{T}_{\mathrm{d}}=\frac{1}{\omega \sqrt{\beta}}
\end{aligned}
$$

The lag correction is:

$$
\mathrm{D}_{\mathrm{si}}=\frac{\mathrm{T}_{\mathrm{i}} \mathrm{s}+1}{\left(10 \mathrm{~T}_{\mathrm{i}} \mathrm{s}+1\right) \mathrm{s}}
$$

Select $\mathrm{Ti}=8 \mathrm{Td}$, then the gyro ring open-loop transfer function is:

$$
\mathrm{G}_{1}=\mathrm{K}_{\mathrm{s}} \mathrm{D}_{\mathrm{sd}} \mathrm{D}_{\mathrm{si}} \mathrm{G}_{0}
$$

Using the MATLAB program to analyze the ring-ring characteristics and closed-loop characteristics of the gyro ring, the system isolation degree is $45.3 \mathrm{~dB}$, and the step response satisfies the oscillation peak requirement, which fully meets the design requirements.

3.3. Simulink tool simulation

The transfer function model and the gyro ring and position loop correction parameters designed for MATLAB software are simulated by Simulink tool. The simulation block diagram is shown in Fig.

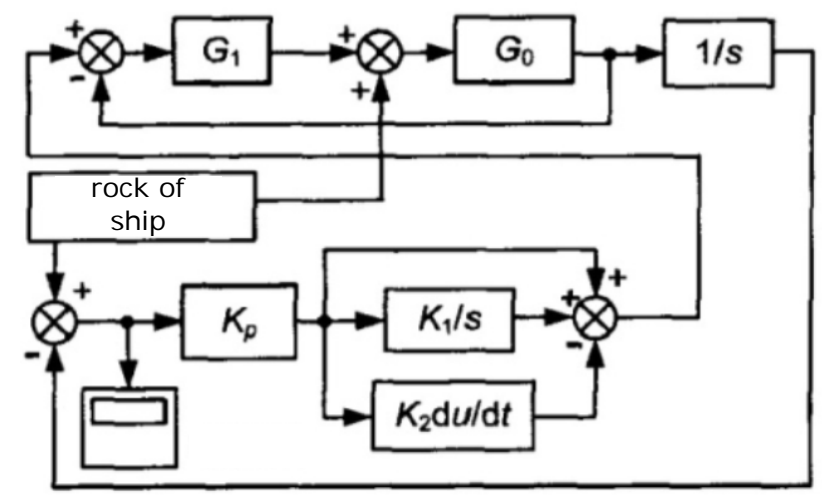

Figure 1. Simulink tool simulation graphics

In Fig. 1, the upper two transfer functions G0 and G1 are the driving chain transfer function and the gyro open-loop transfer function, respectively, and the feedback constitutes the gyro closed loop. The ship shaking signal generator generates a simulated hull swing signal as a given input position loop.

The simulation results are that the stability error peak is less than $0.0052^{\circ}$, that is, the stability accuracy is less than $0.00365^{\circ}$, and the result satisfies the requirement of the index.

\section{Actual test results}

Will be in the simulation block diagram of the transfer function call Simulink tool in the discretization function can be converted into $\mathrm{Z}$ domain function, and then enter it into the software program to form the actual control system. The results are shown in Fig. 


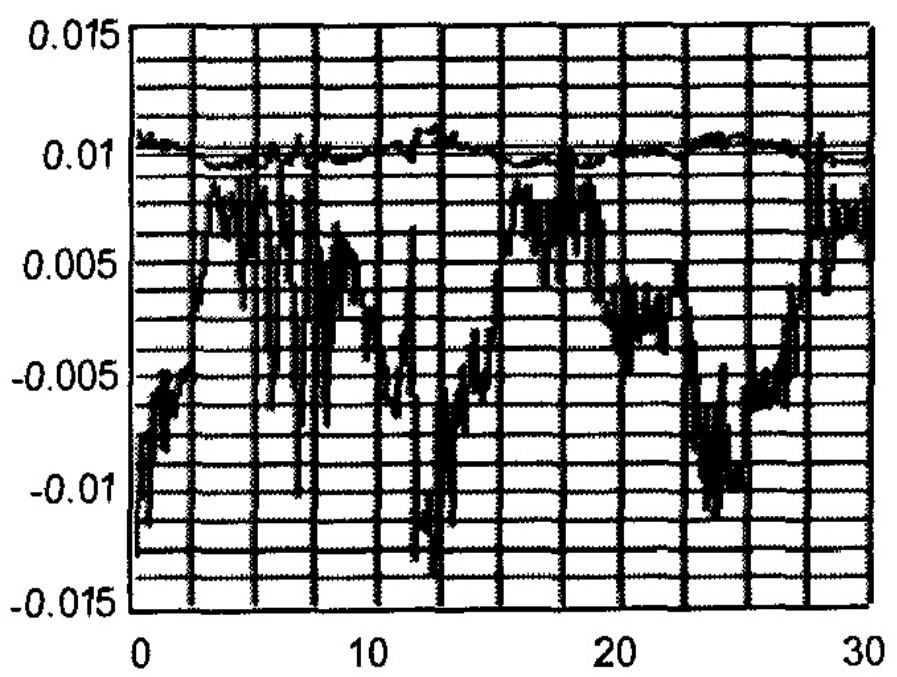

Figure 2. System actual stability effect

The peak of the stability error in Fig. 2 is less than $0.0102^{\circ}$, that is, the stability accuracy is less than $0.00725^{\circ}$. Visible stability accuracy to meet the design requirements.

\section{Summary}

The software simulation results and the actual results are basically the same. It shows that the software can be used to analyze the shipborne antenna control system, and then Simulink simulation tool can be used to evaluate the system performance in advance, which greatly improves the working efficiency and avoids the debugging of the system by using the wrong parameters in practice.

\section{Reference:}

[1] Xue Dingyu. Computer aided design of control system [M]. Beijing: Tsinghua University Press, 2006.

[2] FRANKLIN G F. Automatic control principle and design [M]. Beijing: People's Posts and Telecommunications Press, 2007.

[3] Huang Zhonglin. Control system MATLAB calculation and simulation [M]. Beijing: National Defense Industry Press, 2004. 